\title{
Effect of Airway Management and Impedance Threshold Device on Circulation, Survival and Neurological Outcome in Adult Out-Of-Hospital Cardiac Arrest
}

\author{
David Chase, Angelo Salvucci, Rafael Marino, Robin Shedlosky, \\ Nancy Merman, Katy Hadduck \\ Ventura County Fire Department, Camarillo, CA, USA \\ Email: David.Chase@ventura.org
}

Received 20 January 2014; revised 14 February 2014; accepted 17 March 2014

Copyright (C) 2014 by authors and Scientific Research Publishing Inc.

This work is licensed under the Creative Commons Attribution International License (CC BY). http://creativecommons.org/licenses/by/4.0/

\section{Abstract}

Purpose: This study was designed to study the effect of early use of the King Airway (KA) and impedance threshold device (ITD) in out-of-hospital cardiac arrest on $\mathrm{ETCO}_{2}$ as a surrogate measure of circulation, survival, and cerebral performance category (CPC) scores. After analysis of the first 9 month active period the KA was relegated to rescue airway status. Methods: This was a prospective pre-post study design. Patients $>18$ years with out-of-hospital cardiac caused arrest were included. Three periods were compared. In the first "non active" period conventional AHA 30/2 compression/ventilation ratio CPR was done with bag mask ventilation (BMV). No ITD was used. After advanced airway placement the compression/ventilation ratio was $10 / 1$. In the second period continuous compressions were done. Primary airway management was a KA with an ITD. After placement of the KA the compression/ventilation ratio was 10/1. In the third period CPR reverted to 30/2 ratio with a two hand seal BMV with ITD. CPR ratio was 10/1 post endotracheal intubation (ETI) or KA. The KA was only recommended for failed BMV and ETI. Results: Survival to hospital discharge was similar in all three study periods. In Period 2 there was a strong trend to CPC scores $>2$. The study group hypothesized that the KA interfered with cerebral blood flow. For that reason the KA was abandoned as a primary airway. Comparing Period 1 to Period 3 there was a trend to improved survival in the bystander witnessed shockable rhythm (Utstein) subgroup, particularly if a metronome was used. $\mathrm{ETCO}_{2}$ was significantly increased in Period 2 and trended up in Period 3 when compared to Period 1. Advanced airway intervention had a highly significant negative association with survival. Conclusion: The introduction of an ITD into our system did not result in a statistically significant improvement in survival. The study groups were somewhat dissimilar. $\mathrm{ETCO}_{2}$ trended up. When comparing Period 1 to Period 3, the bundle of care was associated with a trend towards increased survival in the Utstein subgroup, particularly with a me- 
tronome set at 100. Multiple confounders make a definitive conclusion impossible. Advanced airways showed a significant association with poor survival outcomes. The KA was additionally associated with poor neurologic outcomes.

\title{
Keywords
}

\author{
Airway Management; Out-Of-Hospital Cardiac Arrest; Cardiopulmonary Resuscitation; \\ Endotracheal Intubation; Supraglottic Airways; Emergency Medical Services
}

\section{Introduction}

The King Airway (KA) is inserted blindly with no or minimal interruption of chest compressions. The impedance threshold device (ITD) is designed to increase circulation during CPR. This study was initially designed to study the effect of early use of the KA and ITD in out-of-hospital cardiac arrest on end-tidal carbon dioxide $\left(\mathrm{ETCO}_{2}\right)$ as a surrogate measure of circulation, survival, and cerebral performance category (CPC) scores. Our hypothesis was that the combination of uninterrupted compressions and early use of an ITD would increase neurologically intact survival in adult out-of-hospital cardiac arrest patients [1]-[4]. After the first 9 month post (active) study period the KA was relegated to rescue airway status and outcomes were compared vs. actual airway management. The use of the ITD was continued.

\section{Methods}

This was a prospective pre-post study design. All patients 18 years or older with out-of-hospital presumed cardiac caused arrest were included. The study took place in a coastal southern California county with a 2010 census of 823,318 and an area of 2208 square miles.

The study was IRB and State EMS approved. Each segment of the study represented an overall county EMS change in protocol. There was no randomization and no non active treatment group. Each protocol change was within AHA guidelines and felt to represent best practices. Based on the emergent nature of patient presentation and the above parameters consent was waived.

The study spanned from 3/2008 to 8/2012. Results over three periods were compared. In the first (pre 18 month) period protocols included CPR at a 30:2 compression ventilation ratio with bag mask ventilation (BMV), and 10:1 ratio after advanced airway placement. In the second (post 9 month) period protocols included immediate continuous chest compressions, early insertion of the KA (King LTS-D, King Systems) as a primary airway, use of the ITD and 10:1 compression ventilation ratio. In the third period (post 10 - 36 month) period the KA was abandoned as a primary airway. CPR was done with a 30:2 compression to ventilation ratio with two hand seal BMV and 10:1 ratio with endotracheal intubation (ETI) or KA. The airway was managed with BMV with ITD in place. KA was only recommended for failed BMV and ETI. One group in the study (County Fire) used a metronome set at $100 . \mathrm{ETCO}_{2}$ was sampled by a microstream device between the bag and ITD and

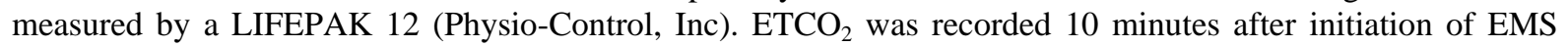
CPR $(X)$ and again 5 and 10 minutes later $(X+5)$ and $(X+10)$. Summary statistics were generated for all periods. Statistical analysis was performed using an unpaired t-test for $\mathrm{ETCO}_{2}$ and two-tailed Fisher's exact for clinical outcome data.

\section{Results}

Overall there were 521 patients in the first period (pre 18 months). 193 had return of spontaneous circulation (ROSC) (37\%), 66 survived to discharge (12.7\%), 60 had CPC score of 1 or 2 (11.5\%) leaving 6 with CPC scores of 3 or $4(1.2 \%)$. In the second period (post 9 months KA as primary airway) there were 291 patients. 110 had ROSC (37.8\%), 32 survived to discharge (11\%), 20 had CPC scores of 1 or 2 (6.9\%) leaving 12 with CPC scores of 3 or 4 (4.1\%). 11 of 12 CPC 3 or 4 survivors had KA. During this study period $37.5 \%$ of survivors were CPC 3 or 4 . Overall there were 865 patients in the third period (post months 10 - 36 KA deleted, ITD continued). 340 had ROSC (39.3\%), 107 survived to discharge (12.4\%), 94 had CPC scores of 1 or 2 (10.9\%) leav- 
ing 13 with CPC scores of 3 or 4 (1.5\%) (Figure 1).

The pre 18 months and post 10 - 36 month subgroups were somewhat dissimilar. 101 of 521 were in bystander witnessed shockable rhythm in the pre 18 month period (19.4\%) vs. 141 of 865 in the third 27 month period (16.3\%). This represents by percentage 19\% less patients in Period 3 in the subgroup, other than EMS witnessed VF/VT, most likely to survive (Figure 2).

Comparing survival of bystander witnessed shockable rhythm (classic Utstein subgroup) from Periods 1 (18 months) to Period 3 (27 months) yields the following: Period 1101 patients of which 59 (58.4\%) had ROSC, 34 (33.7\%) were discharged alive and 33 (32.7\%) had CPC scores of 1 or 2. Period 3141 patients of which 94 (66.7\%) had ROSC, 51 (36.2\%) survived to discharge and 49 (34.8\%) had CPC scores of 1 or 2 (Figure 3).

Comparing survival of bystander witnessed shockable rhythm with the use of a metronome set at 100 from Period 1 to Period 3 yields the following: Period 1, 49 patients of which 28 (57.1\%) had ROSC, 18 (36.7\%) survived to discharge and 17 (34.6\%) had CPC scores of 1 or 2. Period 3, 80 patients of which 56 (70\%) had ROSC, 36 (45\%) survived to discharge and 34 (42.5\%) had CPC scores of 1 or 2 (Figure 4). CPC 1 or 2 outcome difference yields a $\mathrm{P}$ value of 0.18 (not statistically significant).
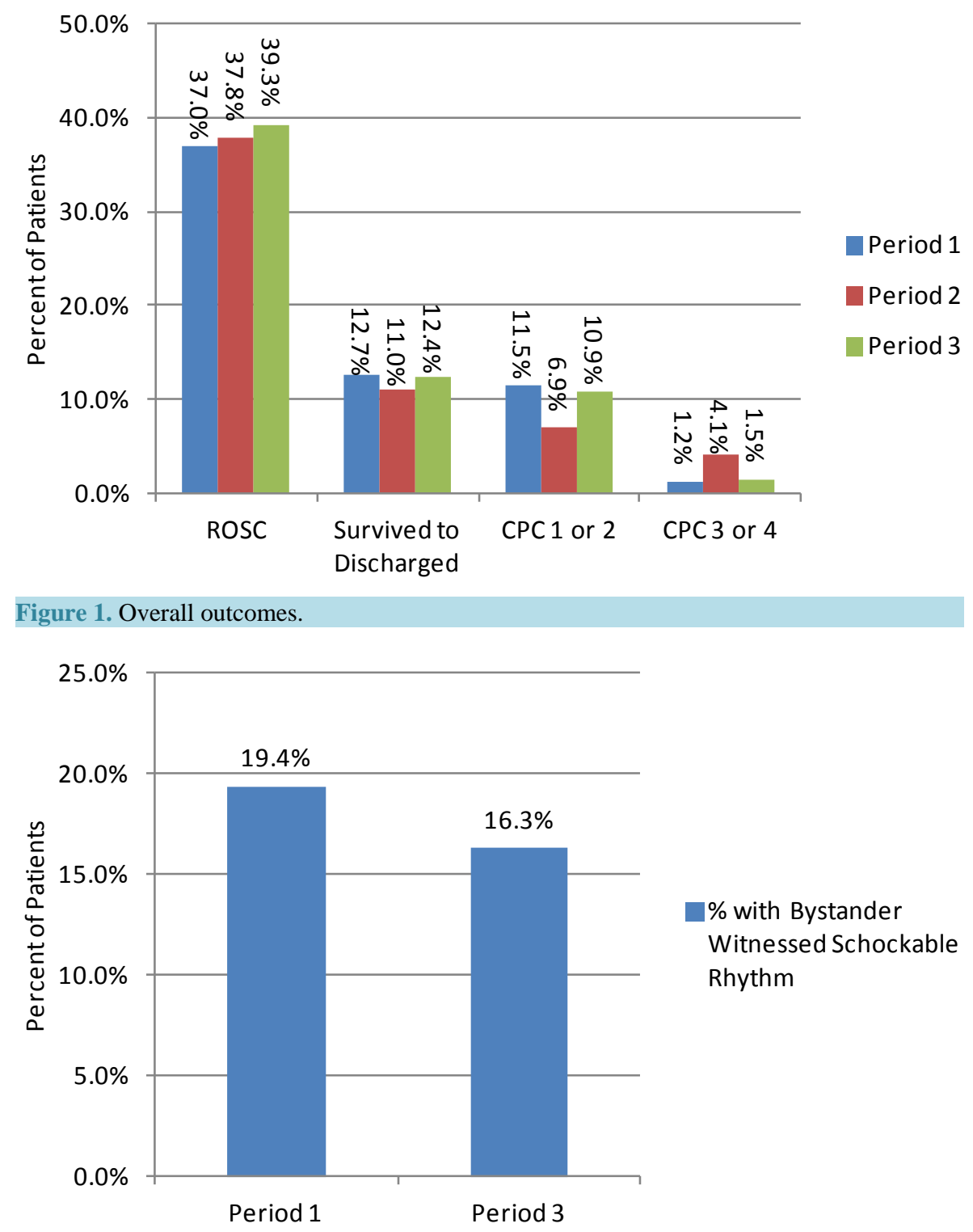

Figure 2. Bystander witnessed shockable rhythm. 


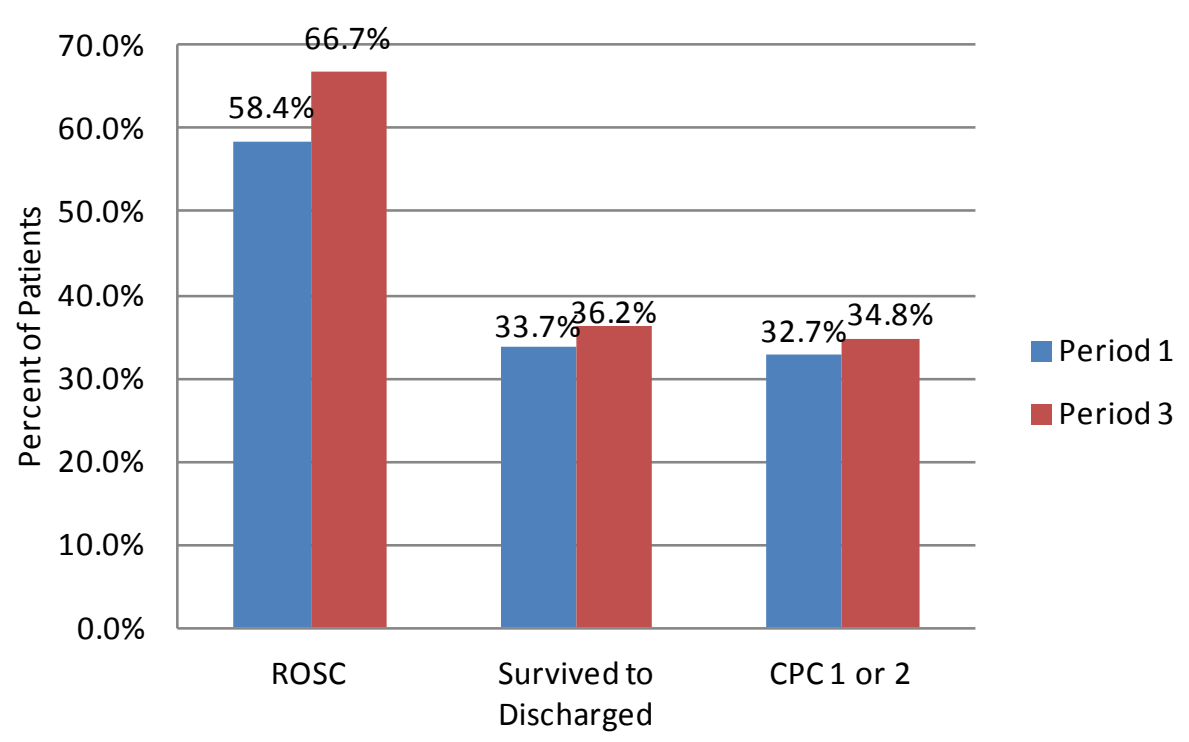

Figure 3. Overall bystander witnessed shockable rhythm.

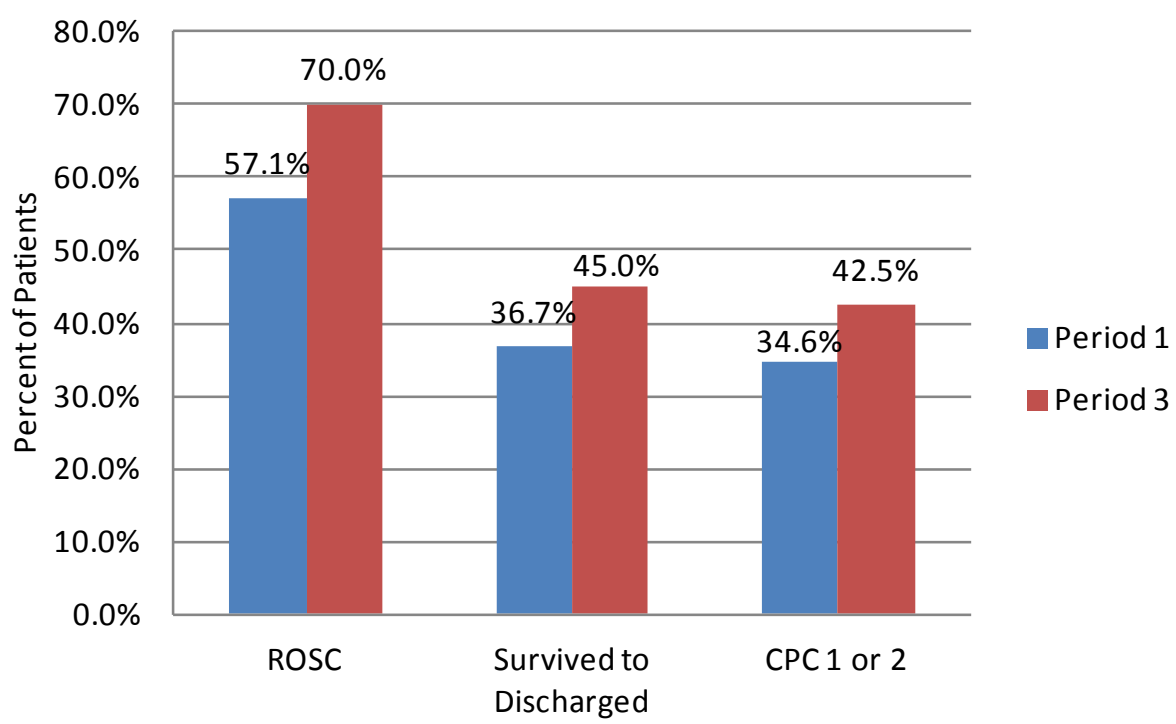

Figure 4. Bystander witnessed shockable rhythm with metronome.

$\mathrm{ETCO}_{2}$ outcomes are depicted in Figure 5. Although there is a trend towards increased $\mathrm{ETCO}_{2}$ in Period 3 vs. Period 1, only Period 2 yielded a statistically significant increase in $\mathrm{ETCO}_{2}$ suggesting that the KA/ITD combination improved systemic circulation. $X$ in Period 2 is statistically greater than in Period $1(P<0.05)$. The $N$ values are low; Period $1 \mathrm{~N}=53$, Period $2 \mathrm{~N}=41$, and Period $3 \mathrm{~N}=86$. All available $\mathrm{ETCO}_{2}$ data was used. In general $\mathrm{ETCO}_{2}$ was measured only after an advanced airway was placed (ETI or KA). Additionally not all agencies made $\mathrm{ETCO}_{2}$ data available. Some of the Period 1 (retrospective) data had been purged.

Examining outcome vs. airway management yields the following: 1677 adult cardiac arrest cases were reviewed during the entire study period (54 months). 527 were handled with BMV with no advanced airway attempt. Of this group 251 (47.6\%) had ROSC, 125 (23.7\%) survived to discharge, 113 (21.4\%) had CPC scores of 1 or 2 leaving 12 (2.3\%) with CPC score of 3 or 4.663 received ETI in the field. Of this group 229 (34.5\%) had ROSC, 45 (6.7\%) survived to discharge, 39 (5.9\%) had CPC scores of 1 or 2 leaving 6 (0.9\%) with CPC scores of 3 or 4.364 received a KA in the field. Of this group 127 (34.8\%) had ROSC, 30 (8.2\%) survived to discharge, 17 (4.7\%) had CPC scores of 1 or 2 leaving 13 (3.6\%) with CPC scores of 3 or 4 . In this group 43\% of survivors had CPC scores of 3 or 4.12 patients received a Combi-tube (CT) in the field. Of this group 1 
(8.3\%) had ROSC and no patient survived to discharge. 111 patients had an advanced airway attempt and then reverted to BMV. Of this group 35 (31.5\%) had ROSC, 5 (4.5\%) survived to discharge, 4 (3.6\%) had CPC scores of 1 or 2 leaving $1(0.9 \%)$ with a CPC score of 3 or 4 (Figure 6). Comparing CPC 1 or 2 outcomes for BMV 113/527 (21.4\%) to all "ALS" airway interventions either completed or attempted 60/1150 (5.2\%) yields a highly statistically significant difference (P Value $<0.0001$ ).

\section{Discussion}

Similarly to the ROC PRIMED [5] outcome, introducing the ITD into our EMS system did not result in an overall increase in survival. However our pre and post subgroups were somewhat dissimilar with the "pre" group having a greater percentage of bystander witnessed shockable rhythm. Looking overall at the bystander witnessed shockable rhythm subgroup there was a weak trend towards improved outcome. Similarly to the ROC PRIMED post hoc analysis looking at bystander VF with compression rate of 100 - 109 [6] the use of the ITD in conjunction with a metronome set at 100 was associated with a stronger trend towards increased survival. The use of a metronome is an inexpensive and practical solution to driving proper rate of compressions by field providers. After many electronic run reviews the authors have noted that compression rates are frequently greater than 120 without a metronome and when a metronome is being used close to the rate set on the metronome. Looking at the post hoc analysis mentioned above [6] rates greater than 120 are detrimental to patient outcome

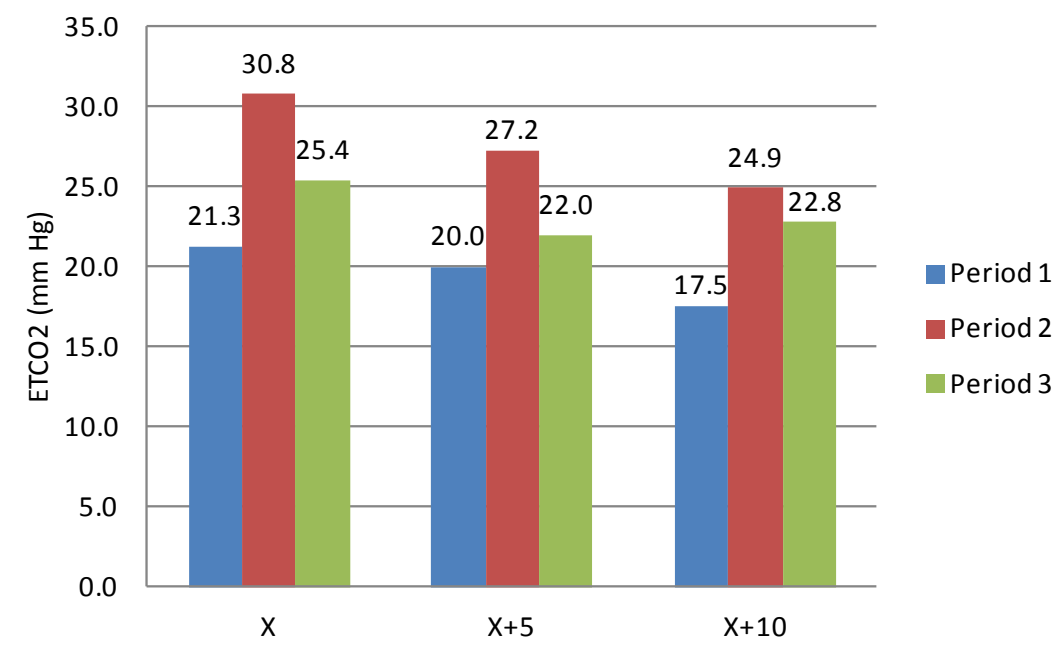

Figure 5. $\mathrm{ETCO}_{2}$ outcomes.

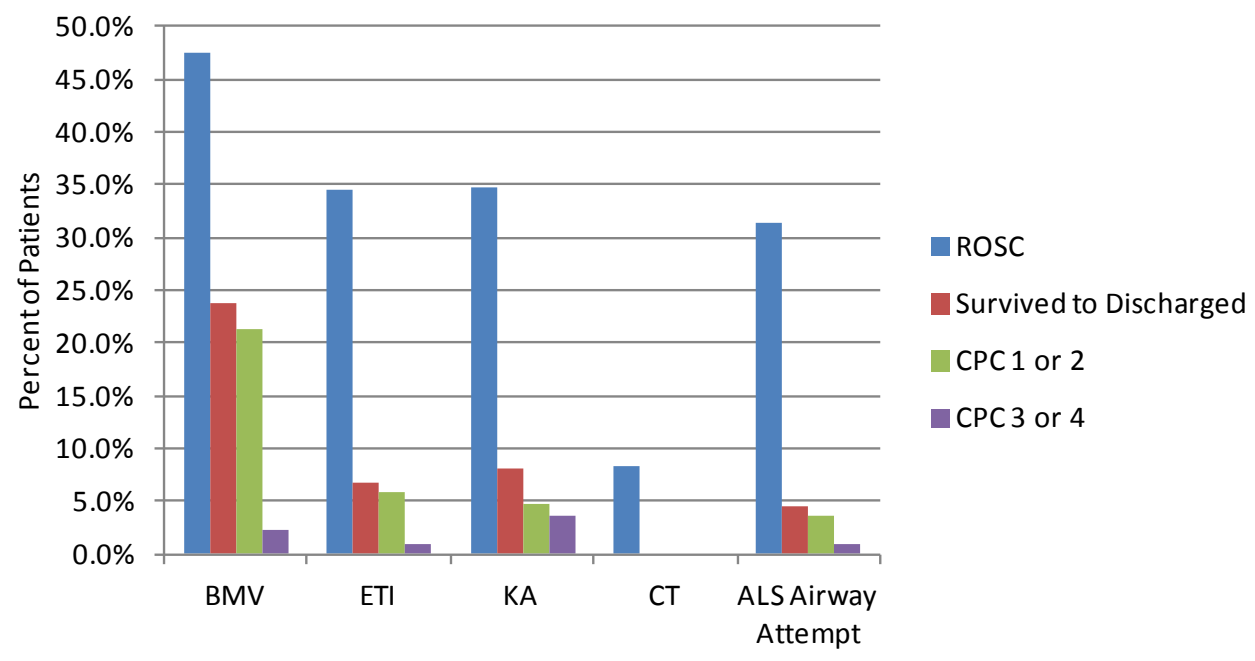

Figure 6. Examining outcomes versus airway management. 
with or without the use of an ITD. During the study period, amiodarone, feedback enhanced CPR training and direction of ROSC patients to STEMI receiving centers with hypothermia was introduced potentially improving outcomes. Outcomes were tabulated in nine month segments. Coincident with feedback enhanced CPR training the second nine month segment of Period 1 had a trend to increased overall survival CPC 1 or 2 with 35 of 279 (13\%) CPC 1 or 2 vs. 25 of $242(10 \%)$ in the first segment. Any improvements in outcome may be secondary to feedback enhanced CPR and improved hospital care (including PCI and hypothermia) rather than the ITD. However the second segment of Period 1 had the highest percentage of bystander witnessed arrest (44.1\%) and bystander witnessed shockable rhythm (20.8\%) of any nine month segment.

The use of a KA as a primary airway was associated with a strong trend towards poor cerebral performance amongst survivors. This led the authors to abandon the KA as a primary airway and to begin to look at the effect on survival of airway choice and to the hypothesis that supraglottic airways may have a detrimental effect on cerebral perfusion. Colbert, et al demonstrated that with a laryngeal mask airway carotid flow was marginally decreased in patients with a normal perfusion state [7]. The authors were concerned that in the low perfusion state of CPR that the effect would be greater. One of the authors was subsequently involved in a porcine study that demonstrated a reduction of carotid flow of $40 \%-75 \%$ with supraglottic airways during CPR [8]. Three subsequent studies [9]-[11] have shown a negative association of advanced airway placement on survival of outof-hospital cardiac arrest patients. As well three studies have shown worse outcomes with SGAD versus ETI [9] [11] [12]. We have noted the same negative association with either the placement or the attempt at placement of an advanced airway. In part this is selection bias. Patients that regain airway reflexes after a single shock do not generally get an advanced airway. This negative association may be beyond selection bias. Many of our cases had a KA placed as a primary airway, eliminating selection bias. Some systems such as Seattle frequently intubate and have excellent outcomes. In our system that runs about 400 cardiac arrests per year utilizing 250 paramedics ETI too frequently results in excessive interruption of compressions. Additionally the placement of an endotracheal tube makes it far too easy to achieve high intrathoracic pressures resulting in decreased perfusion. The authors feel that the KA and many other supraglottic airway devices may have an adverse effect on cerebral perfusion.

\section{Conclusions}

The introduction of an ITD into our system did not result in a statistically significant improvement in overall survival or survival in any subgroup. The study groups were somewhat dissimilar. When comparing the first 18 month period to the last 27 month period, the bundle of care, including amiodarone, optimized hospital care with hypothermia and PCI, and the ITD was associated with a trend towards increased survival in bystander witnessed shockable rhythm particularly when used with a metronome set at 100. However, when comparing the second 9-month segment of the first period (after feedback CPR training, but before the ITD), to the final 27 month period, the ITD without metronome was associated with a trend toward decreased survival. Of note the second 9 month segment of Period 1 had the highest percentage of bystander witnessed arrest and the highest percentage of bystander witnessed VF of any 9 month segment. Multiple confounders make a definitive conclusion impossible.

Advanced airways showed a significant association with poor survival outcomes. The KA was additionally associated with poor neurologic outcomes. We now recommend ETI only if BMV is failing and KA only for failed ETI. We feel that is the proper course for systems that have a high number of ALS providers in comparison to opportunities for ETI.

A metronome or other device should be used to achieve optimal compression rates, especially if an ITD is used. Excellent CPR with near continuous compressions of proper depth, rate, and release is critical and is improved with feedback enhanced CPR training [13].

\section{References}

[1] Bobrow, B.J., et al. (2008) Minimally Interrupted Cardiac Resuscitation by Emergency Medical Services for Out-ofHospital Cardiac Arrest. JAMA, 299, 1158-1165. http://dx.doi.org/10.1001/jama.299.10.1158

[2] Aufderheide, T.P., et al. (2007) A Tale of Seven EMS Systems: An Impedence Threshold Device and Improved CPR Techniques Double Survival Rates After Out-of-Hospital Cardiac Arrest. AHA Scientific Session.

[3] Thigpen, K., et al. (2008) Implementation of the 2005 Cardiopulmonary Resuscitation Guidelines and Use of an Im- 
pedance Threshold Device Improve Survival from Inhospital Cardiac Arrest. Annals of Emergency Medicine, 51, 475. http://dx.doi.org/10.1016/j.annemergmed.2008.01.282

[4] Yannopoulos, D., et al. (2007) Acute Management of Sudden Cardiac Death in Adults Based upon the New CPR Guidelines. Europace, 9, 2-9. http://dx.doi.org/10.1093/europace/eul126

[5] Aufderheide, T.P., et al. (2011) A Trial of an Impedance Threshold Device in Out-of-Hospital Cardiac Arrest. The New England Journal of Medicine, 365, 798-806. http://dx.doi.org/10.1056/NEJMoa1010821

[6] Idris. American Heart Association, Scientific Sessions, Poster 2012.

[7] Colbert, S.A., et al. (1998) The Laryngeal Mask Airway Reduces Flow in the Common Carotid Artery Bulb. Canadian Journal of Anesthesia, 45, 23-27. http://dx.doi.org/10.1007/BF03011987

[8] Segal, N., et al. (2012) Impairment of Carotid Artery Blood Flow by Supragottic Airway Use in a Swine Model of Cardiac Arrest. Resuscitation, 83, 1025-1030. http://dx.doi.org/10.1016/j.resuscitation.2012.03.025

[9] Wang, H.E., et al. (2012) Endotracheal Intubation versus SGA Insertion on OHCA. Resuscitation, 83, 1061-1066. http://dx.doi.org/10.1016/j.resuscitation.2012.05.018

[10] Hasegawa, K., et al. (2013) Association of Prehospital Advanced Airway Management with Neurologic Outcome and Survival in Patients With Out-of-Hospital Cardiac Arrest. JAMA, 309, 257-266. http://dx.doi.org/10.1001/jama.2012.187612

[11] Fouche, P.F., et al. (2013) Airways in Out-of-Hospital Cardiac Arrest: Systematic Review and Meta-Analysis. Prehospital Emergency Care, Early Online.

[12] Tanabe, S., et al. (2013) Comparison of Neurological Outcome between Tracheal Intubation and Supraglottic Airway Device Insertion of Out-of-Hospital Cardiac Arrest Patients. The Journal of Emergency Medicine, 44, 389-397. http://dx.doi.org/10.1016/j.jemermed.2012.02.026

[13] Yeung, J., et al. (2009) The Use of CPR Feedback/Prompt Devices during Training and CPR Performance: A Systematic Review. Resuscitation, 80, 743-751. http://dx.doi.org/10.1016/j.resuscitation.2009.04.012

\section{Abbreviations}

(KA) King Airway; (ITD) impedance threshold device; (CPR) cardiopulmonary resuscitation; (CPC) cerebral performance category; (BMV) bag mask ventilation; (ETI) endotracheal intubation; $\left(\mathrm{ETCO}_{2}\right)$ end-tidal carbon dioxide; (EMS) emergency medical services; (ROSC) return of spontaneous circulation; (CT) Combi-tube; (AHA) American Heart Association; (SGAD) supraglottic airway device. 